\title{
Applications of Parallel-Element, Embedded Mesh-Cap Acoustic Liner Concepts
}

\author{
M. G. Jones* and D. M. Nark ${ }^{\dagger}$ \\ NASA Langley Research Center, Hampton, VA 23681 \\ A. Baca ${ }^{\ddagger}$ and C. R. Smith $^{\S}$ \\ Hexcel Corporation, Casa Grande, AZ 85130
}

\begin{abstract}
This study explores progress achieved with 2DOF, 3DOF, and MDOF acoustic liners constructed with mesh caps embedded within a honeycomb core. These liner configurations offer potential for broadband noise reduction, and are suitable for conventional aircraft implementation. Samples for each configuration are tested in the NASA normal incidence tube and grazing flow impedance tube, with and without a wire mesh facesheet. Impedances based on these measured data compare favorably with those predicted using a transmission line impedance prediction model. Predicted impedances are then used as input for an aeroacoustic propagation code to compute axial acoustic pressure distributions in the grazing flow tube. These predicted distributions compare favorably with the corresponding measured distributions at frequencies away from the frequency of peak attenuation, but suffer slight degradation for frequencies very near the peak attenuation frequency, where the predicted results are sensitive to input impedance changes. As expected, the noise reduction frequency range increases as more degrees of freedom are included. Although the specific results achieved herein may differ from those that would be achieved with other 2DOF, 3DOF, and MDOF liners, this comparison highlights some of the key features that can be exploited in the design of parallel-element, embedded mesh-cap liners.
\end{abstract}

\section{Introduction}

In response to increasingly stringent aircraft noise requirements imposed by the International Civil Aviation Organization and the Federal Aviation Administration, the NASA Advanced Air Vehicles Program seeks to develop more effective noise reduction concepts suitable for implementation on conventional aircraft. Of particular interest to the current study is propulsion noise, consisting of contributions due to the jet and fan, which is dominant for the takeoff condition and is similar in magnitude with airframe noise for the approach condition. ${ }^{1}$ Due in large part to fan geometry modifications ${ }^{2,3}$ and the increased usage of high bypass-ratio engines, other noise sources have been sufficiently reduced such that broadband fan noise has become a major source of objectionable noise for communities near airports.

Current aircraft employ acoustic liners mounted in the interior walls of engine nacelles, in the inlet or the aft-bypass duct, to reduce this broadband fan noise. One option is to use a single-degree-of-freedom liner (SDOF, see Fig. 1-a) with a microperforate facesheet. However, a more common broadband liner consists of two air layers separated by a porous septum, with a conventional perforated facesheet, and is typically labeled as a two-degree-of-freedom liner (2DOF, see Fig. 1-b). Whereas each of these configurations has heretofore allowed aircraft manufacturers to achieve the current noise constraints, further fan noise reductions will require more novel configurations.

* Senior Research Scientist, Research Directorate, Structural Acoustics Branch, AIAA Associate Fellow.

$\dagger$ Senior Research Scientist, Research Directorate, Structural Acoustics Branch, AIAA Associate Fellow.

¥Senior Design Engineer, Research and Technology Division.

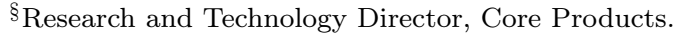




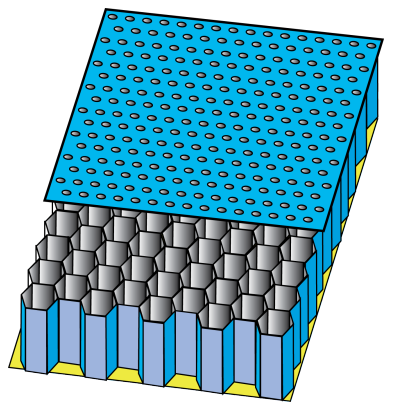

(a) SDOF acoustic liner.

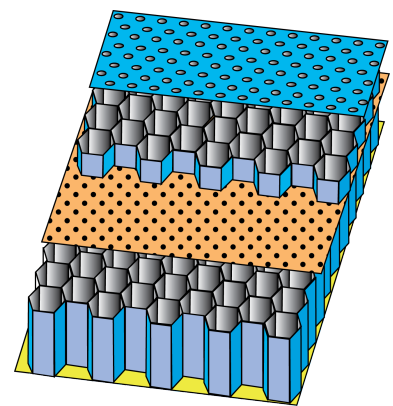

(b) 2DOF acoustic liner.

Figure 1: Sketch of conventional SDOF and 2DOF acoustic liners.

Over the last three decades, the NASA Liner Physics Team has explored a variety of conventional and unconventional acoustic liner concepts for reduction of broadband fan noise. Significant effort has been expended in the study of smeared-impedance liners ${ }^{4-7}$ that consist of core chambers with variable depths, all covered by a conventional perforate. These variable-depth chambers are placed in close proximity to each other, such that their combined effect is to achieve a desired impedance spectrum that is effectively uniform over their surface. By replicating these groups of variable-depth chambers, the entire liner can be made to achieve this desired impedance spectrum that has been tuned to provide broadband noise reduction. As an extension of this concept, NASA has also investigated configurations for which the chamber depth is varied along the axial extent of the liner. This depth variation may be continual ${ }^{8-10}$ or segmented (e.g., multisegment liner). ${ }^{11,12}$

Three other configurations considered by NASA are porous honeycomb, ${ }^{13,14}$ dual-resonance, ${ }^{15}$ and embedded-membrane ${ }^{16}$ liners. The porous honeycomb liner is a conventional perforate-over-honeycomb liner for which the honeycomb walls are sufficiently porous to support sound transmission between adjacent core chambers. When combined with the sound transmission in the main waveguide (e.g., in the flow duct), this causes the liner to behave as an extended-reaction, bulk liner, and to therefore provide broadband absorption. The dual-resonance liner consists of a Helmholtz resonator embedded within a quarter-wavelength resonator. Each of these resonators can, to a large extent, be independently tuned to allow broadband absorption. The embedded-membrane liner contains a membrane within each honeycomb chamber. By careful selection of the height and physical properties (tension, thickness, etc.) of the embedded membrane, these liners can also be tuned to achieve broadband absorption.

With the exception of the porous honeycomb concept, these liner concepts are currently dependent on continued advances in additive manufacturing (3D printing). Indeed, additive manufacturing is advancing very rapidly and provides hope that some of these concepts will be suitable for flight applications in the relatively near future.

Yet another option to achieve broadband noise reduction uses mesh caps ${ }^{17}$ mounted within each individual honeycomb chamber to achieve a controlled surface impedance spectrum. Whereas the aforementioned liner concepts are not yet ready for flight applications, this last option is suitable for such application. This is of great interest to the NASA Liner Physics Team, as we have been tasked with the development of liner configurations for near-term evaluation in an upcoming flight test.

The purpose of the current paper is to review progress in the design, implementation, and evaluation of multi-degree-of-freedom (MDOF) acoustic liners constructed with mesh caps. The impedance prediction model and aeroacoustic propagation code are briefly described in Section II. Section III provides a brief review of results from an earlier study, ${ }^{6}$ and Section IV documents key improvements in the fabrication process for mesh-cap liners that have occurred since that study. Section V provides a description of the test liners and the two acoustic waveguides used to evaluate them. Key results are discussed in Section VI, and some concluding remarks are offered in Section VII. 


\section{Analysis}

\section{A. Impedance Prediction Model}

The impedance prediction model used in this study is the Wave Propagation Model (WPM). This transmission line analysis assumes the individual chambers of the liner are comprised of one or more layers. Two layer types are considered - a finite thickness of air and a mesh cap with constant DC flow resistance. Since the WPM has been discussed in detail in earlier papers, ${ }^{5,6}$ the current discussion is limited to an overview of the general mechanisms of the model. To aid in this discussion, figure 2 provides a sketch of four chambers of a liner core, for which each chamber contains one mesh cap. As indicated in the sketch, the location (height within chamber) of each mesh cap may be unique, and the different colors are meant to convey the possibility that each mesh cap may have a unique DC flow resistance.

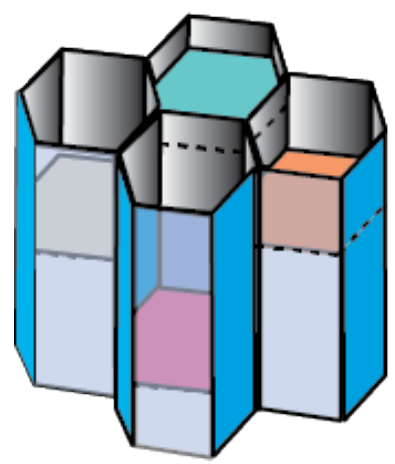

Figure 2: Sketch of four chambers (cells) of a liner core.

The analysis is initiated by assuming the backplate to be rigid and impervious to sound. Changes in the acoustic pressure, $p$, and particle velocity, $u$, across each layer of an individual chamber are then computed via transmission coefficients that depend on the type of layer being considered (air gap or wire mesh). This process is repeated (across each layer) until the acoustic pressure and particle velocity are determined at the surface of the core chamber (just below the facesheet, if one exists) of the selected chamber. For the current study, each test liner is evaluated with and without a wire mesh facesheet. When the wire mesh facesheet is included, it is simply treated as an additional layer in the transmission line analysis. The result of these calculations is a predicted surface impedance, $\zeta_{c h}$, for the individual chamber. This process is conducted for each chamber of the full liner.

The surface impedance spectra of the individual chambers are then combined to compute the effective impedance across the liner surface. For this computation, it is preferable to use admittance $\left(\beta_{c h}=1 / \zeta_{c h}\right)$. The effective admittance across a selected extent of liner surface is given by

$$
\beta_{s}=\Omega \sum_{i=1}^{N_{c h}} \beta_{c h, i}
$$

where $N_{c h}$ represents the number of chambers that combine to form the liner and $\Omega$ is the surface open area ratio (porosity) of the liner. The uniform, effective, surface impedance of the liner is then given by $\zeta_{s}=1 / \beta_{s}$. It has been demonstrated that the surface impedance variability can be ignored (impedance can be assumed uniform) for local-reacting, variable-impedance liners if the impedance variability is uniformly distributed and is confined within an extent that is less than one-third of a wavelength ${ }^{11}$ of the source frequency.

\section{B. Propagation Code}

The CHE aeroacoustic propagation code used in this study has been discussed in detail in an earlier study. ${ }^{18}$ This code employs a finite element method based on the convected Helmholtz equation to predict the complex acoustic pressure field throughout the grazing flow impedance tube (GFIT), which is the flow duct used in 
this study. For the purposes of this study, the flow profile is assumed to be uniform, and the liner is assumed to be installed on the upper wall (as in the GFIT). Also, the 2D version of the code is used, i.e., only plane waves are supported in the hardwall sections upstream and downstream of the duct. The CHE code includes the effects of reflections at the leading and trailing edges of the liner, and has the potential to capture reflections at the duct termination. For the purposes of this study, the duct termination is assumed to be anechoic. The effects of this assumption, while not overly restrictive, will become evident in the results to follow.

\section{Review of 2012 Results}

Some of the earlier NASA research with mesh-cap liners was presented in $2012,{ }^{6}$ and included results for 2-layer and 3-layer liners. These liners were evaluated using the NASA Langley normal incidence tube (NIT), and thus neglected the effects of mean flow over the liner surface. No facesheets were included for these liners, as the main focus of the study was to investigate the suitability of the WPM to predict the effects of the embedded mesh caps.

Figure 3 presents comparisons of predicted and measured impedance spectra for one of the 2-layer liners. The comparison is quite acceptable (note the expanded scale) for the normalized resistance (real component of impedance), and is better for the normalized reactance (imaginary component of impedance). It should be noted that impedances are normalized by the quantity $\rho c$, where $\rho$ and $c$ are the density and sound speed in air at ambient conditions, throughout this paper.

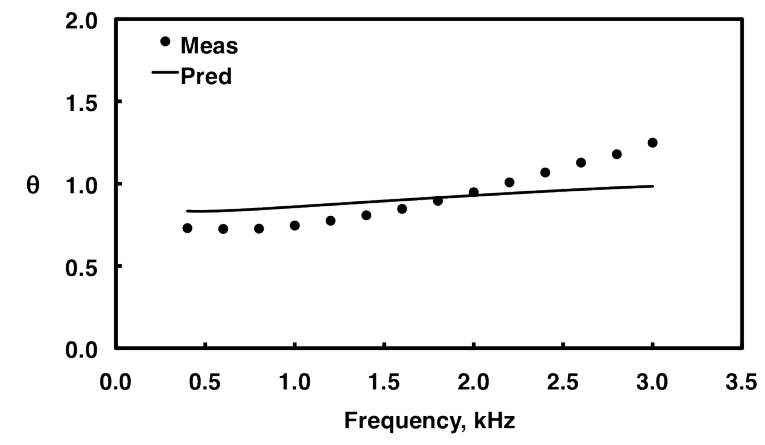

(a) Normalized resistance, 2-layer core.

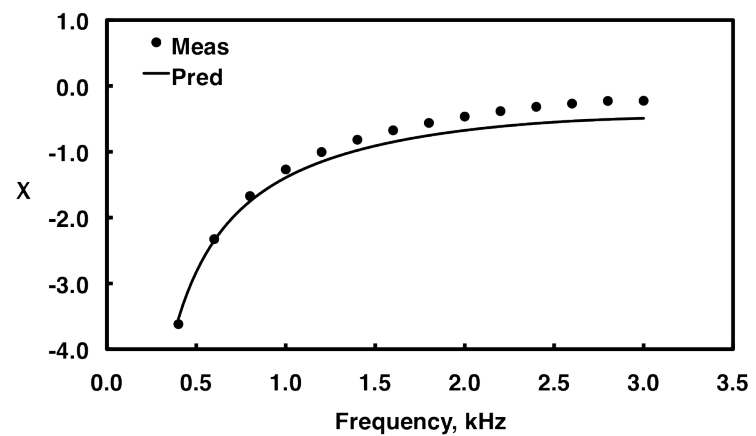

(b) Normalized reactance, 2-layer core.

Figure 3: Normalized impedance spectra for 2-layer liner core.

One possible explanation for the differences between the predicted and measured impedances has to do with the inability of the WPM to account for the shape of the mesh cap. As shown in figure 4, each mesh cap has a parabolic shape, whereas the WPM assumes the mesh layer to be perfectly flat. This results in a variable length for the air layers above and below each mesh cap across the span of the honeycomb chamber. Thus, in order to improve the comparison between the predicted and measured impedance spectra, either the model needs to be extended to account for parabolic-shaped mesh caps, or the fabrication process needs to be altered such that the mesh caps are indeed flat.

Given the fabrication processes used in 2012, it was also difficult to maintain precise control of the meshcap DC flow resistance. Although some broadband liner configurations are less sensitive to these effects, that is not always the case. Thus, it was also deemed important to improve this portion of the process. 


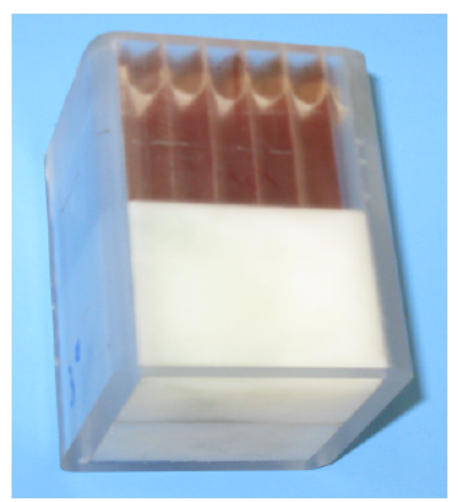

Figure 4: Photo of 2DOF acoustic liner with an embedded mesh cap and no facesheet.

\section{Fabrication Process Improvements}

The fabrication process for these types of liners has continued to improve. Due to changes in the mounting technique (i.e., the manner in which mesh caps are bonded to the walls of the honeycomb chamber), the mesh caps are now nearly flat across the entirety of the chamber. Also, advances in the fabrication process now allow improved control over the effective (as installed) mesh-cap DC flow resistance.

The sample shown in figure 4 was fabricated using Hexcel's Acousti-Cap ${ }^{\circledR}$ product. In order to improve the uniformity of the Acousti-Cap ${ }^{\circledR}$ product, Hexcel has evolved the cap design over time. The original Acousti-Cap ${ }^{\circledR}$ (and the second-generation Bias-Cap) design used a precision dipping process to both lock the mesh septa in place, as well as tune the mesh DC flow resistance by closing part of the mesh area with resin. When combined with the need to allow for MDOF liners that consist of multiple unique chambers, this made the manufacturing process more complicated.

The most recent design, Hybrid-Cap, allows the resistance of each cap to be tuned using the geometrical design of the cap rather than having to rely on the dipping process. This not only results in a more uniform 2DOF liner, but also opens up possibilities to create more complex, next generation liners. Hybrid-Cap combines the PEEK (polyetheretherketone) acoustic mesh with a PEEK film such that the film acts as a window frame carrier to hold the mesh in place in the honeycomb, hence the specific acoustic resistance can be tuned by varying the window frame size. Using this concept, the PEEK film carrier can be modified to achieve such designs as $3 \mathrm{DOF}$ and $\mathrm{MDOF}$ on a production scale, without requiring the manufacturing process to be more complicated.

\section{Experimental Method}

Three liner configurations (2DOF, 3DOF, and MDOF) are considered in the current study. These liners were initially designed for other studies that will be presented in future papers. The current study will focus on the capability to predict the impedance spectra and corresponding sound absorption of each configuration. Each of these liner configurations is fabricated in two formats, one sized for installation in the NIT and the other sized for installation in the GFIT. This allows the effects of mean flow, source sound pressure level (SPL), and facesheet configuration to be independently evaluated.

\section{A. Test Liners}

Figure 5 provides sketches of the three liner configurations. Each sketch depicts two adjacent chambers of a liner. Dotted lines are used to depict the septa or facesheet, where the septa are mesh-caps as described in Section IV and the facesheet is a layer of wire mesh. The labels indicate the target DC flow resistances $\left(R_{f}\right.$, in MKS Rayls) for each mesh, and the dimensions are provided along the right edge. These liners are constructed using a modular approach (facesheet is clamped, not bonded, to the liner core) such that the effects of other facesheets (e.g., conventional perforate) can also be evaluated. Results will be provided for 
each of these liner configurations, both with and without the wire mesh facesheet.

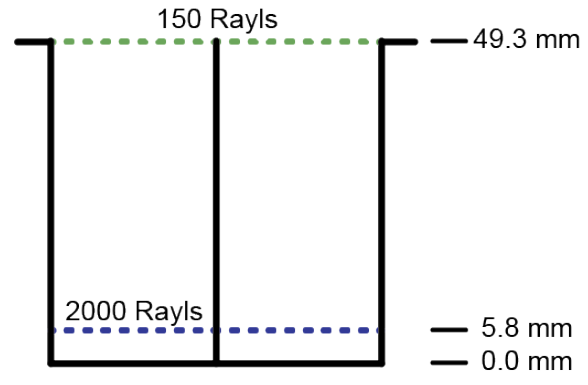

(a) 2DOF configuration (TL1).

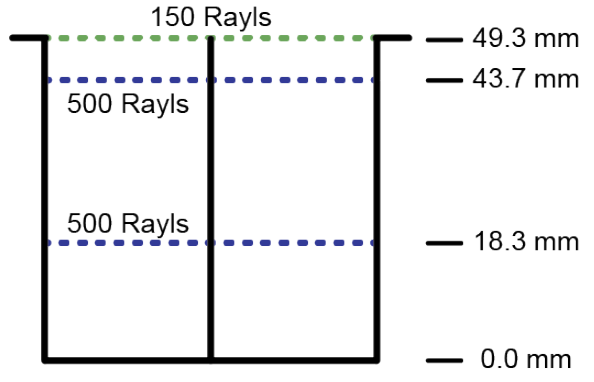

(b) 3DOF configuration (TL2).

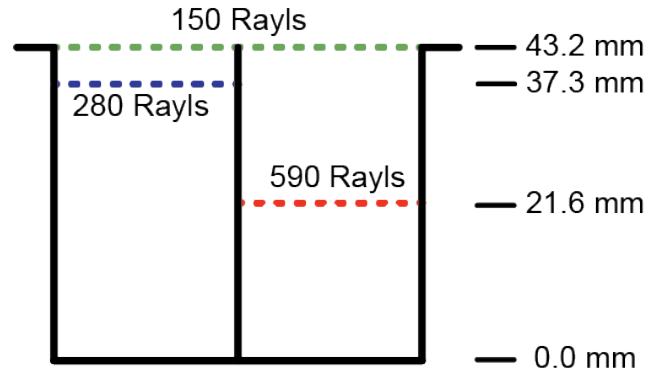

(c) MDOF configuration (TL3).

Figure 5: Sketches of test liners.

The first liner (labeled TL1) is a 2DOF configuration, i.e., each chamber contains one embedded mesh cap and every chamber is identical. Given the high resistance of the mesh cap (2000 MKS Rayls; normalized flow resistance of 5), very little sound is expected to be transmitted into the lower air layer next to the rigid back plate. As such, this liner is expected to exhibit the acoustic characteristics of an SDOF liner with a core depth of $43.5 \mathrm{~mm}$.

The TL2 liner is a 3DOF configuration. Each chamber contains two mesh caps, and is identical to every other chamber in the liner. The two embedded mesh caps have identical $R_{f}$ values (500 MKS Rayls), and are positioned 18.3 and $43.7 \mathrm{~mm}$ from the back plate.

The TL3 liner is an MDOF configuration. Strictly speaking, all of the liners considered in this study could be labeled as MDOF, as they all provide multiple degrees of freedom. For the purposes of this study, the MDOF label is used to indicate that the TL3 liner is comprised of two unique 2DOF chambers, each of which contains one mesh cap, and these two chambers are assumed to be replicated (in pairs) to form the entire liner. The $R_{f}$ values for the mesh caps in each chamber are different (280 and 590 MKS Rayls, respectively).

Figure 6 provides photographs of each liner. The 2DOF photograph is inverted to show the mesh that is embedded near the back plate (not shown). The end view of the 3DOF liner shows that mesh caps are embedded at two heights, one near the upper surface (where the facesheet would be placed) and the other closer to the bottom (where the back plate would be placed). As indicated above, the MDOF liner consists of two unique chambers. The first is shown in the end row (partially open), where the mesh is embedded near the bottom of the liner core. In the second row, a different mesh is embedded near the surface. The third row (and every alternating row) appears empty because the mesh is embedded too deep in the chamber to be seen. It should be noted that these liner configurations were designed to take advantage of the ability to better control the position and DC flow resistance of the embedded mesh caps. 


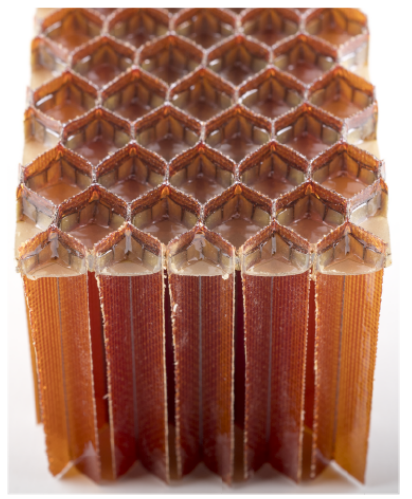

(a) 2DOF configuration (TL1); inverted.

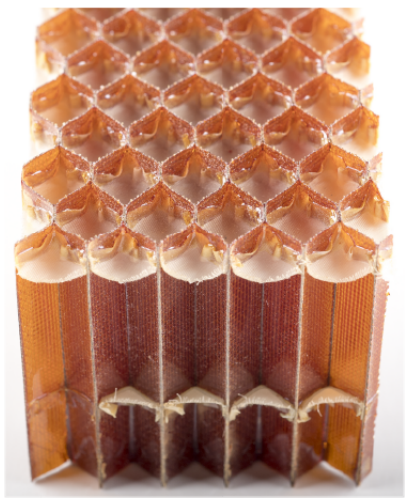

(b) 3DOF configuration (TL2).

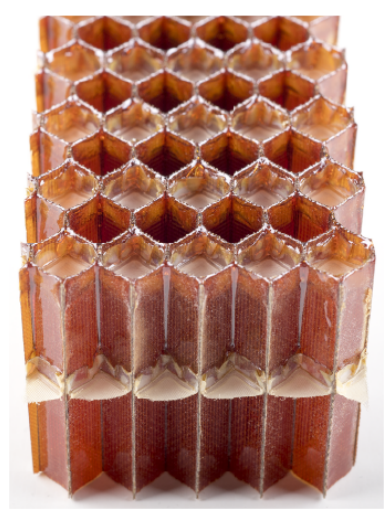

(c) MDOF configuration (TL3).

Figure 6: Photographs of test liners.

\section{B. Normal Incidence Tube Measurements}

The NASA Langley normal incidence tube (NIT) is a $50.8 \mathrm{~mm} \times 50.8 \mathrm{~mm}$ waveguide (see sketch in Fig. 7) that contains six $120-\mathrm{W}$ compression drivers to generate a plane-wave sound field that impinges on the surface of the liner and combines with reflections from the liner to create a standing wave pattern. The Two-Microphone Method (TMM) ${ }^{19,20}$ is used to measure the complex acoustic pressures at two prescribed distances from the liner surface, such that the frequency dependence of the no-flow acoustic impedance of the liner can be computed. Tonal tests (one frequency at a time) are conducted for source frequencies of 0.4 to $3.0 \mathrm{kHz}$ in $0.2 \mathrm{kHz}$ increments, with source sound pressure levels (SPLs) of 120 and $140 \mathrm{~dB}$ at the reference microphone. These tones are generated by a computer-controlled function generator. Two amplitudes are used so that test liner nonlinearities can be evaluated by determining whether the impedance is a function of the reference SPL.

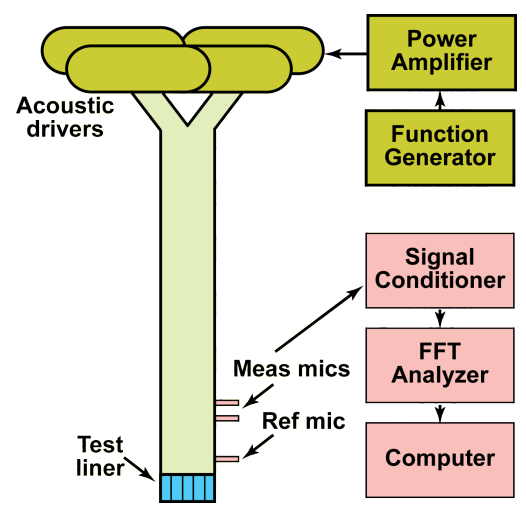

Figure 7: Sketch of NASA normal incidence tube (NIT).

\section{Grazing Flow Impedance Tube Measurements}

The NASA Langley grazing flow impedance tube (GFIT, see Fig. 8) has a cross-sectional geometry of $50.8 \mathrm{~mm}$ wide by $63.5 \mathrm{~mm}$ high, and allows evaluation of acoustic liners with lengths from $50.8 \mathrm{~mm}$ to $609.6 \mathrm{~mm}$ (558.8 $\mathrm{mm}$ length for the liners used in this study). The surface of the test liner forms a portion of the upper wall of the flow duct. Twelve acoustic drivers form an upstream (exhaust mode) source section. For this study, these drivers are used to generate tones (one frequency at a time) over a frequency range of 0.4 
to $3.0 \mathrm{kHz}$ in $0.2 \mathrm{kHz}$ increments, at source levels (peak total SPL measured near the leading edge of the liner) of 120 and $140 \mathrm{~dB}$, and at centerline Mach numbers of 0.0 and 0.3 . Fifty-three (53) microphones are flush-mounted in the lower wall (opposite the liner) of the GFIT, and are used to measure the acoustic pressure field over the axial extent of $1016 \mathrm{~mm}$ (beginning $203.2 \mathrm{~mm}$ upstream of the liner leading edge).

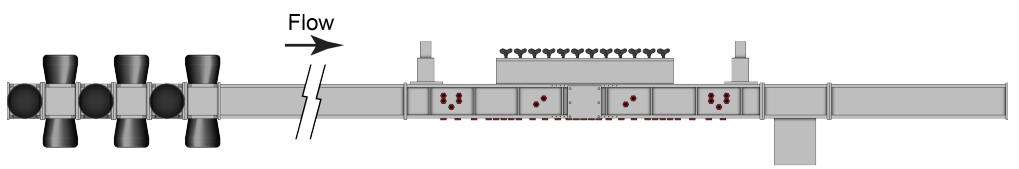

Figure 8: Sketch of NASA grazing flow impedance tube (GFIT).

The Kumaresan and Tufts (KT) algorithm ${ }^{21}$ is used with the measured acoustic pressures to educe the impedance of the liner. Specifically, the axial wavenumber, $K_{n}$, is determined from the acoustic pressures measured with the microphones on the wall opposite the uniform-impedance liner. The unknown impedance, $\zeta$, of the test liner is then computed as

$$
\zeta=\frac{i k}{\lambda_{n}}\left(1-K_{n} M / k\right)^{2} \cot \left(\lambda_{n} H\right), \quad \lambda_{n}^{2}=\left(k-K_{n} M\right)^{2}-K_{n}^{2}
$$

where $i, M, k$, and $H$ are the unit imaginary number $(\sqrt{-1})$, uniform flow Mach number, freespace wavenumber, and duct height, respectively.

\section{Results and Discussion}

The Wave Propagation Model (WPM) is used to predict the impedance spectra for each of the three liner configurations at each of the test conditions. The NIT is used to measure the impedance for each of the small $(50.8 \mathrm{~mm} \times 50.8 \mathrm{~mm})$ samples, and the GFIT is used to educe the impedance for the corresponding larger $(50.8 \mathrm{~mm} \times 558.9 \mathrm{~mm}$ ) samples. Figure 9 provides a comparison of predicted and measured (or educed) impedance spectra for tests conducted at Mach 0.0 with a $120 \mathrm{~dB}$ source. These results are acquired with no facesheet (i.e., only the liner cores are tested). In this and successive figures, the solid and dashed black lines represent the predicted normalized acoustic resistance and reactance spectra, respectively. The red circles and blue diamonds represent the corresponding impedances based on data acquired in the NIT and GFIT, respectively. For these measured results, the solid and open symbols represent the normalized acoustic resistance and reactance (real and imaginary components of impedance), respectively.

For the 2DOF liner, the comparison of the predicted impedance spectra with the corresponding spectra measured in the NIT is quite good. The corresponding GFIT results diverge somewhat at the lowest frequencies, but generally compare favorably with the predicted and NIT results. For the 3DOF liner, the GFIT results track the predicted impedances better than those measured with the NIT. Whereas the two measured results (NIT and GFIT) tend to collapse together, their comparison with the predicted results is less favorable. All three acoustic resistance spectra (predicted, NIT, and GFIT) compare very favorably for the MDOF liner, whereas the reactance spectra diverge at the lower frequencies. Nevertheless, the comparisons are quite acceptable.

Figure 10 provides the corresponding impedance spectra for the configurations where a wire mesh facesheet has been mounted onto each liner. For the 2DOF and 3DOF liners, the effect of adding the 150 MKS Rayls wire mesh facesheet is exactly as expected, i.e., the normalized resistance increases by approximately 0.33 while the normalized reactance is relatively unaffected. The effect of adding the wire mesh facesheet is different for the MDOF liner. Whereas the normalized resistance increases by the expected amount (both predicted and measured), the predicted normalized reactance spectrum shifts to the right. In other words, the predicted resonance frequency shifts from $1900 \mathrm{~Hz}$ with no facesheet to $2500 \mathrm{~Hz}$ with the addition of the facesheet. This is hypothesized to be due to the fact that the MDOF liner consists of 


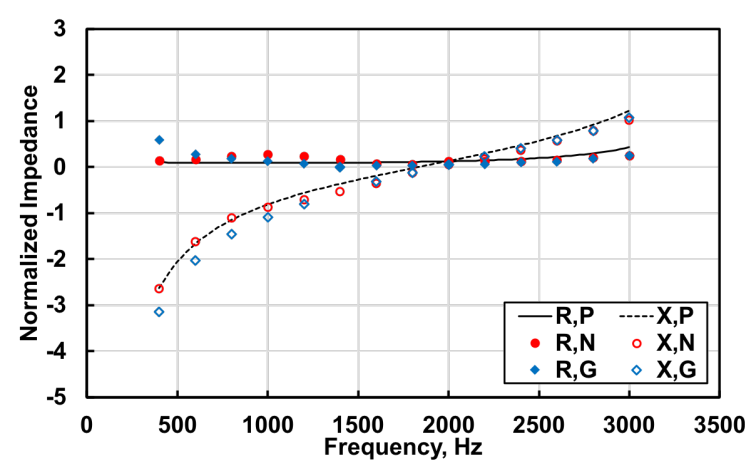

(a) 2DOF (TL1) liner.

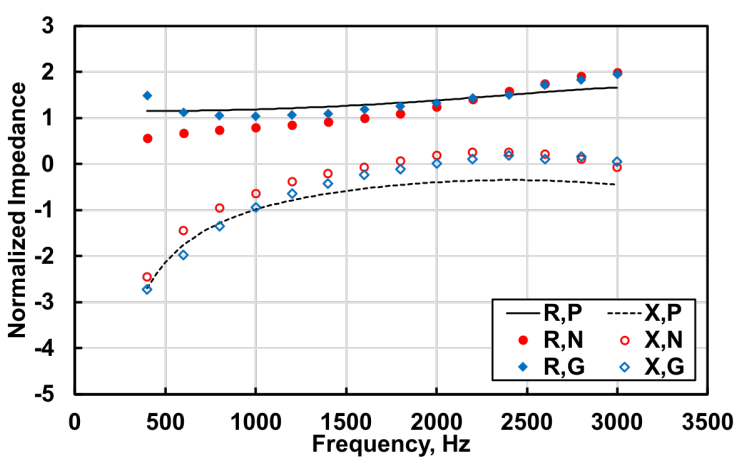

(b) 3DOF (TL2) liner.

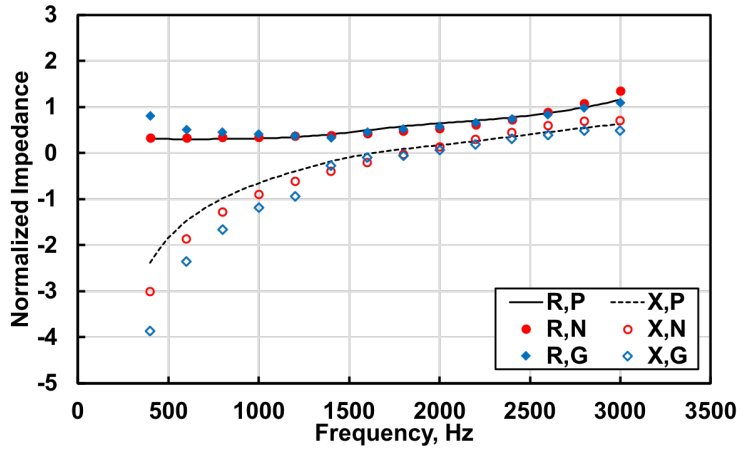

(c) MDOF (TL3) liner.

Figure 9: Predicted and measured impedance spectra; Mach 0.0, $120 \mathrm{~dB}$ source, no facesheet.

combinations of two unique chambers while the 2DOF and 3DOF liners each consist of one unique chamber that is replicated to fill the volume. This change in impedance between adjacent chambers for the MDOF liner has an effect on the resultant predicted impedance. This feature is intended to be the focus of a future study.

Figure 11 demonstrates the impact of these differences in the predicted and measured impedances on the corresponding comparison of predicted and measured axial acoustic pressure distributions for the 2DOF liner with no facesheet. Results are provided for frequencies of 1.2 to $2.2 \mathrm{kHz}$, to encompass the regime where the liner provides the greatest effect on the acoustic pressure field within the GFIT. Figure 11a provides a comparison of sound pressure levels (SPL) measured in the GFIT (solid lines) against those predicted using the CHE propagation code (dashed lines) at frequencies of 1.2, 1.4, and $1.6 \mathrm{kHz}$. Although the impedances compare favorably at these frequencies, the axial acoustic pressure distributions deviate quite significantly. Recall that the CHE is configured (for this study) to assume an anechoic termination (hence, the predicted constant SPL in the hardwall section at the end of the duct). Also observe that the measured attenuation is quite significant (over $60 \mathrm{~dB}$ at $1.6 \mathrm{kHz}$ ). For the next three frequencies (1.8 to $2.2 \mathrm{kHz}$ ), the comparisons are markedly improved (see Fig. 11b). These results clearly indicate that extreme accuracy is needed in order to model the effects of the liner at frequencies where significant attenuation occurs.

Corresponding predicted and measured (only for GFIT) impedance spectra are provided in Figure 12 for the test condition of Mach 0.3 with a source SPL of $120 \mathrm{~dB}$. As expected, the wire mesh facesheet is very insensitive to mean flow effects. Thus, the results are observed to be very similar to those for the Mach 0.0 condition (Fig. 10). It should perhaps be noted that, for each liner included in this study, the results achieved with a $140 \mathrm{~dB}$ source are nearly identical to those achieved with the $120 \mathrm{~dB}$ source. This is to be expected, since the wire mesh facesheet (or the lack of a facesheet) is very linear (insensitive to acoustic 


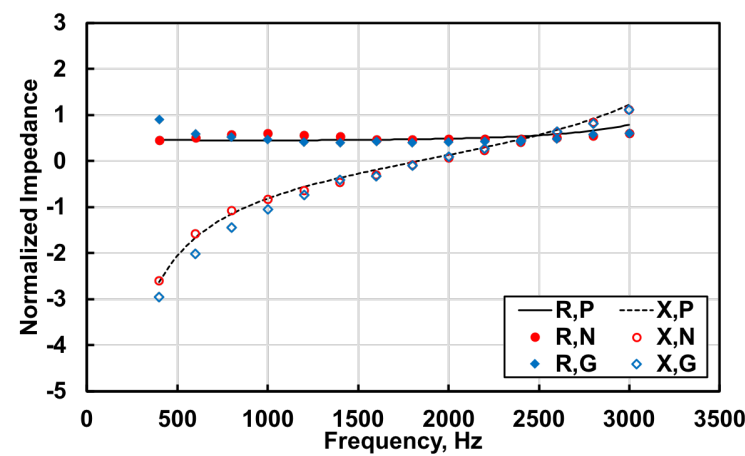

(a) 2DOF (TL1) liner.

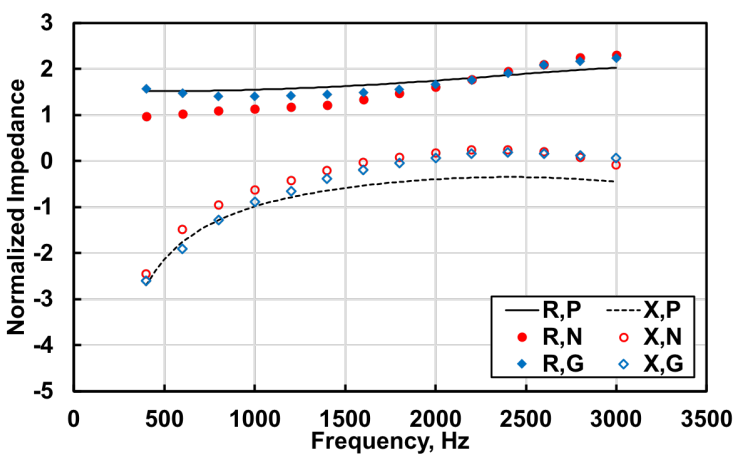

(b) 3DOF (TL2) liner.

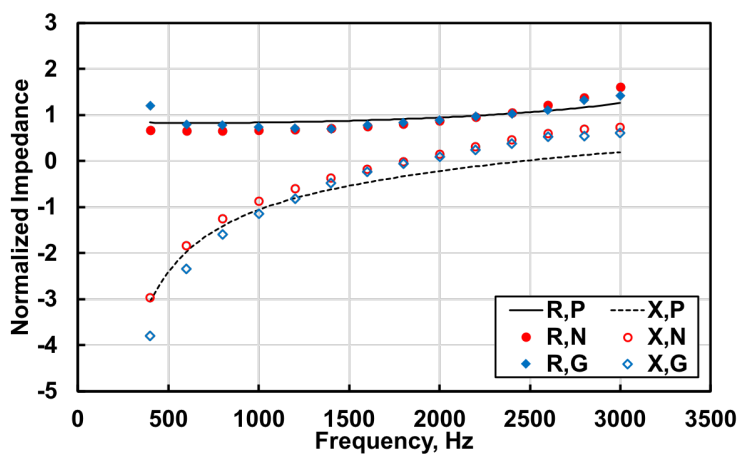

(c) MDOF (TL3) liner.

Figure 10: Predicted and measured impedance spectra; Mach 0.0, $120 \mathrm{~dB}$ source, wire mesh facesheet.

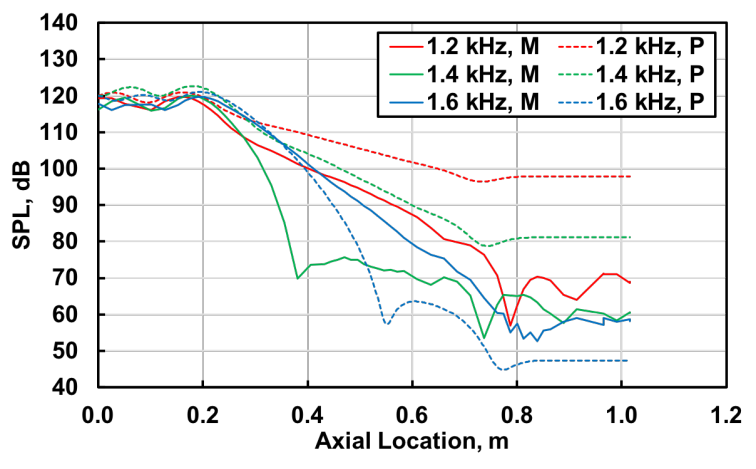

(a) Frequencies of $1.2,1.4$, and $1.6 \mathrm{kHz}$.

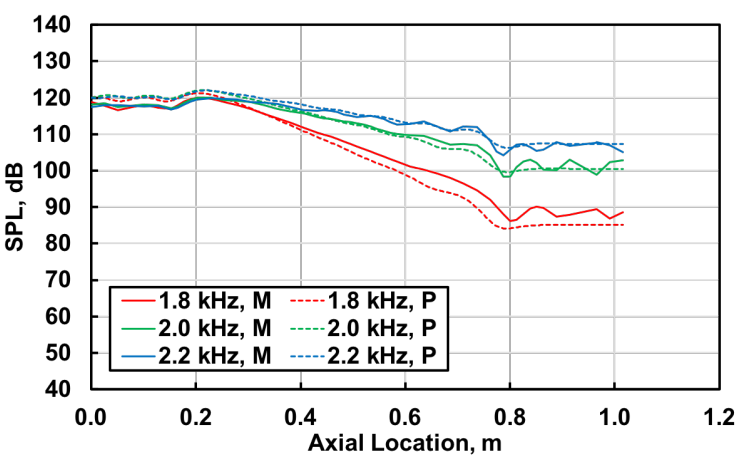

(b) Frequencies of 1.8, 2.0, and $2.2 \mathrm{kHz}$.

Figure 11: Measured and predicted axial acoustic pressure distributions; 2DOF (TL1) liner, Mach 0.0, $120 \mathrm{~dB}$ source, no facesheet. 
particle velocity and, hence, to SPL). For this reason and for the sake of brevity, the results presented in this paper are limited to those achieved with a $120 \mathrm{~dB}$ source.

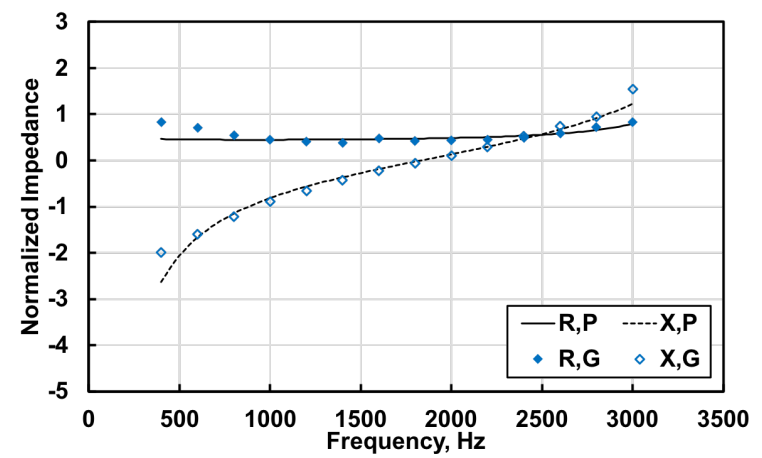

(a) 2DOF (TL1) liner.

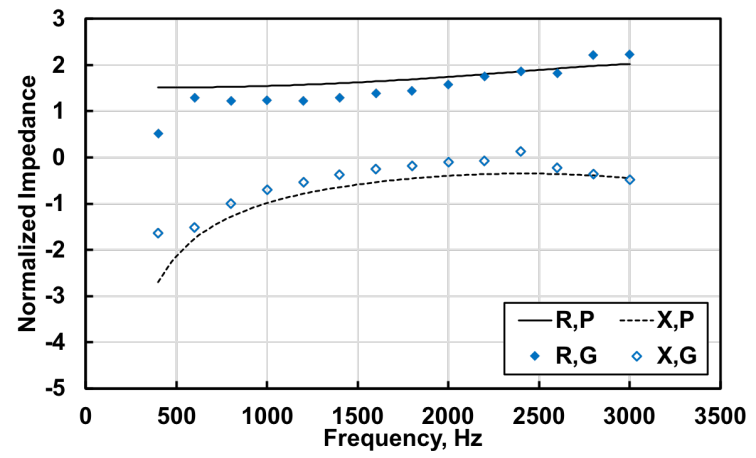

(b) 3DOF (TL2) liner.

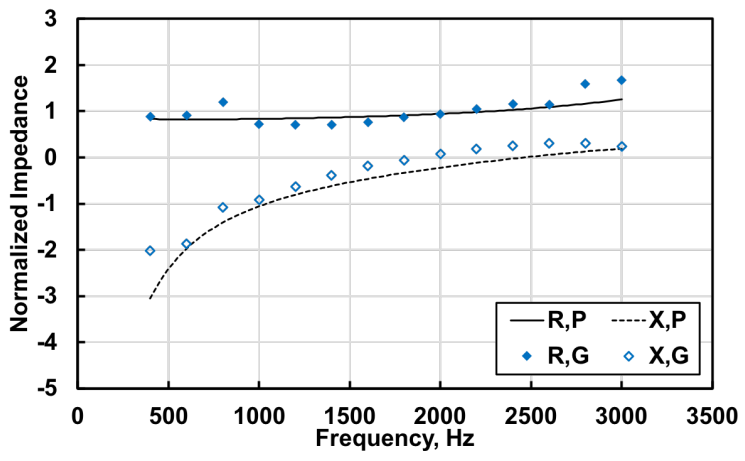

(c) MDOF (TL3) liner.

Figure 12: Predicted and measured impedance spectra; Mach 0.3, $120 \mathrm{~dB}$ source, wire mesh facesheet.

Figure 13 provides axial acoustic pressure distributions for each liner with a wire mesh facesheet. The effects of standing waves due to the termination are evident in the measured results, whereas the prediction assumes the termination to be anechoic. In spite of these differences, the comparisons are quite favorable. The corresponding results for frequencies of 1.2 to $1.6 \mathrm{kHz}$ are also improved (relative to the Mach 0.0 test condition), but not quite as much.

It is perhaps of interest to review the overall acoustic performance of each of these liners. Figure 14 provides the measured attenuation spectra that correspond to the impedance and axial acoustic pressure distributions presented above. In these plots, the red squares, blue circles, and green diamonds represent the attenuations achieved with the $2 \mathrm{DOF}, 3 \mathrm{DOF}$, and MDOF liners, respectively. The attenuation, A, is computed as

$$
\mathrm{A}=\mathrm{SPL}_{\text {source }}-\mathrm{SPL}_{\text {exit }}
$$

where $\mathrm{SPL}_{\text {source }}$ and $\mathrm{SPL}_{\text {exit }}$ represent the sound pressure levels measured at the source $(\mathrm{x}=0.0 \mathrm{~mm})$ and exit $(\mathrm{x}=1016 \mathrm{~mm})$ planes, respectively.

When no facesheet is employed, the 2DOF liner provides attenuation over a very narrow frequency range. Recall that the DC flow resistance for the mesh cap installed near the bottom of the 2DOF liner is quite high (2000 MKS Rayls). This high resistance inhibits sound transmission through the mesh, thereby causing 


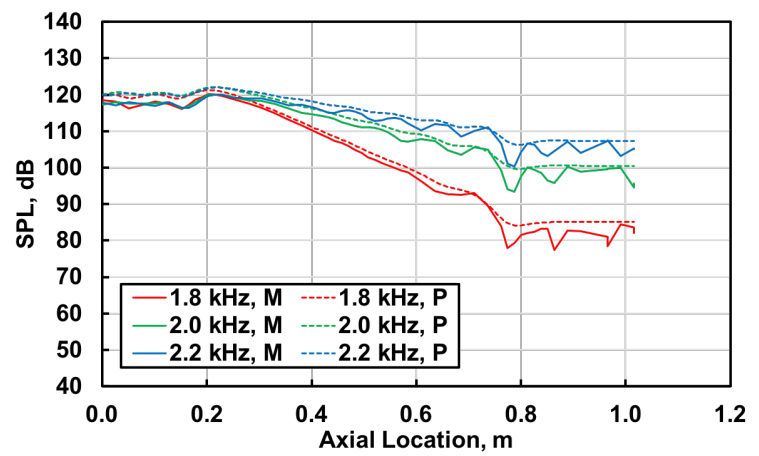

(a) 2DOF (TL1) liner.

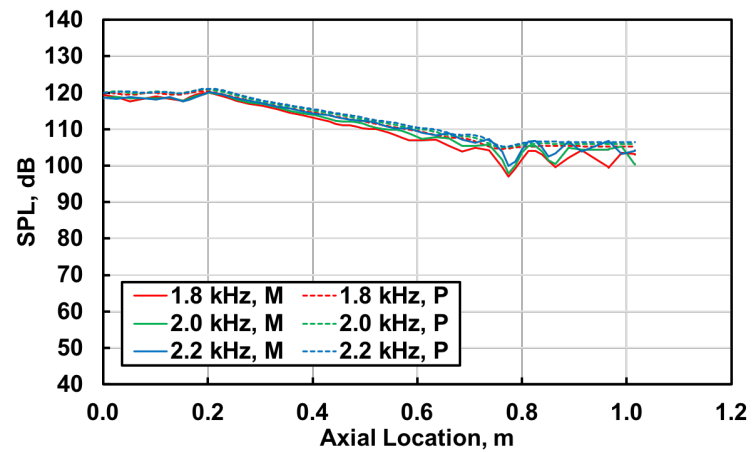

(b) 3DOF (TL2) liner.

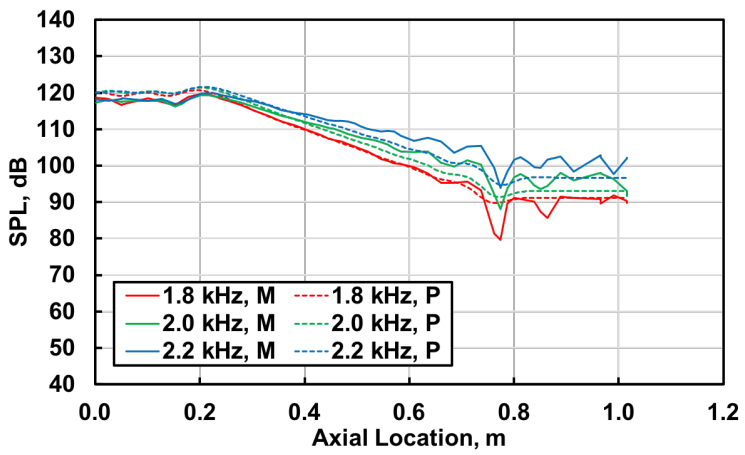

(c) MDOF (TL3) liner.

Figure 13: Measured and predicted axial pressure distributions; Mach 0.3, $120 \mathrm{~dB}$ source, wire mesh facesheet.

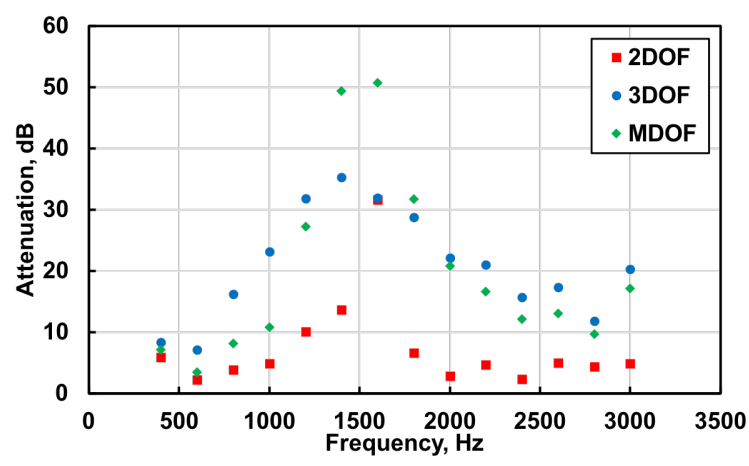

(a) No facesheet; Mach 0.0, $120 \mathrm{~dB}$ source

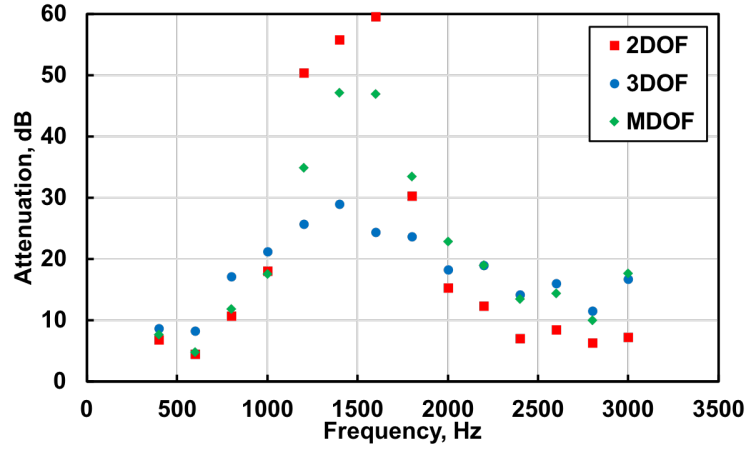

(b) Wire mesh facesheet; Mach 0.0, $120 \mathrm{~dB}$ source.

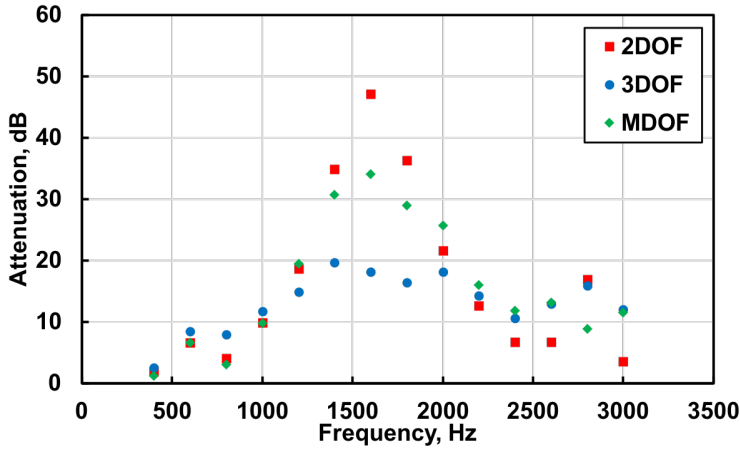

(c) Wire mesh facesheet; Mach 0.3, $120 \mathrm{~dB}$ source.

Figure 14: Measured attenuation spectra; $\mathrm{A}=\mathrm{SPL}_{\text {source }}-\mathrm{SPL}_{\text {exit }}$. 
the liner to behave much like a conventional single-layer liner (i.e., high attenuation over narrow frequency range). The 3DOF and MDOF liners provide much better attenuation across the majority of the frequency range used in this test. This is in large part due to the fact that the 3DOF and MDOF liner cores provide much more resistance at their surface than the 2DOF liner core.

When the wire mesh facesheet is added, the attenuation achieved with the 2DOF liner is significantly improved. The addition of the 150 MKS Rayl facesheet clearly moves the surface impedance toward optimum for the GFIT. A slight decrease in the peak attenuation is observed for the 3DOF and MDOF liners, but otherwise the spectra are quite similar to those achieved without a facesheet. When the flow is turned on (Mach 0.3), the attenuation spectra are generally reduced for all three liners. Nevertheless, they all provide significant broadband attenuation.

It is important to recognize that these results are not necessarily representative of those that would be achieved with other 2DOF, 3DOF, and MDOF liners. The liners used in this study were designed for different applications, each of which had a unique metric that was targeted in the design methodology. Nevertheless, this comparison highlights some of the key features that can be exploited in the design of parallel-element, embedded mesh-cap liners. Also, the Wave Propagation Model is demonstrated to be a useful tool for predicting the acoustic impedance spectra measured with 2DOF, 3DOF, and MDOF acoustic liners manufactured using this design approach.

\section{Concluding Remarks}

This study has explored progress in the design, implementation, and evaluation of 2DOF, 3DOF, and MDOF acoustic liners constructed with mesh caps (resistive sheets) embedded within a honeycomb core. These liner configurations offer the potential for more effective broadband noise reduction, and are suitable for implementation on conventional (or unconventional) aircraft.

Two test samples were fabricated for each liner configuration, one sized to fit the NASA normal incidence tube (NIT) and the other sized to fit the NASA grazing flow impedance tube (GFIT). Each sample was tested in the NIT and GFIT both with and without a wire mesh facesheet (GFIT tests with flow only conducted with facesheet installed), and the impedance spectra based on these data were compared with spectra predicted using a transmission line impedance prediction model (Wave Propagation Model - WPM). In general, impedances predicted using the WPM compare favorably with those based on measured data.

Axial acoustic pressure distributions in the GFIT were computed with the CHE aeroacoustic propagation code using impedances predicted with the WPM as input. These comparisons demonstrate the sensitivity of the propagation code to the input impedance at frequencies near the frequency of peak attenuation. Away from this highly sensitive frequency regime, the predicted and measured acoustic pressure distributions compare favorably.

Based on GFIT results, the attenuation for these three liners is generally as expected. With no facesheet, the 2DOF liner core provides attenuation over a very narrow frequency range, whereas the 3DOF and MDOF liners provide much better attenuation over a wide frequency range. When the wire mesh facesheet is added, the 2DOF liner provides much better attenuation. A slight decrease in the peak attenuation is observed for the 3DOF and MDOF liners, but otherwise the addition of the facesheet has minimal effect. When the flow is turned on (Mach 0.3), the attenuation spectra are generally reduced for all three liners. Nevertheless, they all provide significant broadband attenuation.

Again, it is important to recognize that these results are not necessarily representative of those that would be achieved with other 2DOF, 3DOF, and MDOF liners. Nevertheless, this comparison highlights some of the key features that can be exploited in the design of parallel-element, embedded mesh-cap liners.

\section{Acknowledgements}

This work was funded by the Advanced Air Transport Technology Project of the NASA Advanced Air Vehicles Program, and was conducted as part of a Space Act Agreement (SAA1-21586) between NASA Langley Research Center and Hexcel Corporation. The authors would like to express appreciation to Brian Howerton, Martha Brown, Alonso Reid, and Larry Becker for their efforts in the acquisition of the NIT and GFIT data. 


\section{References}

${ }^{1}$ Lockard, D. P. and Lilley, G. M., "The Airframe Noise Reduction Challenge," NASA TM 213013, 2004.

${ }^{2}$ Envia, E. E., Huff, D. L., and Morrison, C. R., "Analytical assessment of stator sweep and lean in reducing rotor-stator tone noise," AIAA Paper 1996-1791, May 1996.

${ }^{3}$ Woodward, R. P., Elliott, D. M., Hughes, C. E., and Berton, J. J., "Benefits of Swept-and-Leaned Stators for Fan Noise Reduction," Journal of Aircraft, Vol. 38, No. 6, 2001, pp. 1130-1138.

${ }^{4}$ Wirt, L. S., "Analysis, Testing, and Design of Lined Ducts," Journal of the Acoustical Society of America, Vol. 51, No. 5, 1972, pp. 1448-1463.

${ }^{5}$ Parrott, T. L. and Jones, M. G., "Parallel-Element Liner Impedances for Improved Absorption of Broadband Sound in Ducts," Noise Control Engineering Journal, Vol. 43, No. 6, November - December, 1995.

${ }^{6}$ Jones, M. G., Howerton, B. M., and Ayle, E., "Evaluation of Parallel-Element, Variable-Impedance, Broadband Acoustic Liner Concepts," AIAA Paper 2012-2194, June 2012.

${ }^{7}$ Howerton, B. M., Jones, M. G., and Buckley, J. L., "Development and Validation of an Interactive Liner Design and Impedance Modeling Tool," AIAA Paper 2012-2197, June 2012.

${ }^{8}$ Parrott, T. L., Abrahamson, A. L., and Jones, M. G., "Measured and Calculated Acoustic Attenuation Rates of Tuned Resonator Arrays for Two Surface Impedance Distribution Models With Flow," NASA TP 2766, January 1988.

${ }^{9}$ Jones, M. G., Watson, W. R., Nark, D. M., and Howerton, B. M., "Evaluation of a Variable-Impedance Ceramic Matrix Composite Acoustic Liner," AIAA Paper 2014-3352, June 2014.

${ }^{10}$ Jones, M. G., Watson, W. R., Nark, D. M., and Howerton, B. M., "Evaluation of Variable-Depth Liner Configurations for Increased Broadband Noise Reduction," AIAA Paper 2015-2697, June 2015.

${ }^{11}$ Jones, M. G., Watson, W. R., Nark, D. M., Schiller, N. H., and Born, J. C., "Optimization of Variable-Depth Liner Configurations for Increased Broadband Noise Reduction," AIAA Paper 2016-2783, May 2016.

${ }^{12}$ Jones, M. G., Nark, D. M., Watson, W. R., and Howerton, B. M., "Variable-Depth Liner Evaluation Using Two NASA Flow Ducts," AIAA Paper 2017-3022, June 2017.

${ }^{13}$ Parrott, T. L. and Jones, M. G., "Cascaded Perforates as One-Dimensional, Bulk Absorbers," AIAA Paper 2006-2402, 2006.

${ }^{14}$ Jones, M. G. and Parrott, T. L., "Assessment of Bulk Absorber Properties for Multi-Layer Perforates in Porous Honeycomb Liners," AIAA Paper 2006-2403, 2006.

${ }^{15}$ Beck, B. S., Schiller, N. H., and Jones, M. G., "Impedance assessment of a dual-resonance acoustic liner," Applied Acoustics Journal, Vol. 93, June 2015, pp. 15-22.

${ }^{16}$ Chambers, A., Manimala, J. M., and Jones, M. G., "Improved Low-Frequency Broadband Absorption using 3D Folded Cavity Acoustic Liners," to appear in the proceedings of the Institute of Noise Control Engineering NoiseCon17 Congress and Conference, Grand Rapids, MI, June, 2017. 1, June 2017.

${ }^{17}$ Syed, A. A. and Ichihashi, F., "The Modeling and Experimental Validation of the Acoustic Impedance of Multi-Degreesof-Freedom Liners," AIAA Paper 2008-2927, May 2008.

${ }^{18}$ Watson, W. R., Jones, M. G., and Parrott, T. L., "Validation of an Impedance Eduction Method in Flow," AIAA Journal, Vol. 37, No. 7, July 1999, pp. 818-824.

${ }^{19}$ Chung, J. Y. and Blaser, D. A., "Transfer function method of measuring in-duct acoustic properties: I. Theory," Journal of Acoustical Society of America, Vol. 68, 1980, pp. 907-921.

${ }^{20}$ Jones, M. G. and Parrott, T. L., "Evaluation of a multi-point method for determining acoustic impedance," Journal of Mechanical Systems and Signal Processing, Vol. 3, No. 1, 1989, pp. 15-35.

${ }^{21}$ Watson, W. R., Carpenter, M. H., and Jones, M. G., "Performance of Kumaresan and Tufts Algorithm in Liner Impedance Eduction with Flow," AIAA Journal, Vol. 53, No. 4, April 2015, pp. 1091-1102. 